\title{
La coevaluación y autoevaluación en la asignatura de Distribución de la Información Espacial del MUIGG en la UPV
}

\author{
Eloina Coll Aliaga ${ }^{a}$, José Carlos Martinez-LLario ${ }^{\text {a }}$ \\ a Departamento de Ingeniería Cartográfica, Geodesia y Fotogrametría (ecoll@cgf.upv.es), \\ (jomarlla@cgf.upv.es)
}

\begin{abstract}
Resumen
En la evaluación de la asignatura de Distribución espacial del Máster Universitario en Ingeniería en Geomática y Geoinformación (MUIGG) de la Universitat Politècnica de València se utilizan, entre otras técnicas las de coevaluación y autoevaluación. Se pretende con ello evaluar parte de las competencias especificas y transversales que el estudiante debe alcanzar en la asignatura. Con la autoevaluación, el estudiante reflexiona de lo que está aprendiendo y hace que sea capaz de reconocer sus fortalezas y debilidades permitiendo que se responsabilice de mejorar su propio proceso de aprendizaje. En la coevaluación los compañeros se valoran entre ellos y este tipo de evaluación permite que los estudiantes sean más activos en clase, Ellos deben ser capaces de expresar juicios críticos sobre sus compañeros y recibir una retroalimentación que les ayude a mejorar en su aprendizaje. En este artículo se muestran los procesos seguidos en clase con las temporizaciones y rubricas utilizadas, así como los resultados obtenidos en los dos últimos cursos académicos
\end{abstract}

Palabras clave: Coevaluación, autoevaluación, evaluación, aprendizaje colaborativo.

\section{Introducción}

En este trabajo se presenta la metodología utilizada como parte de evaluación de la asignatura de Distribución de la Información Espacial, asignatura troncal del Máster Universitario en Ingeniería en Geomática y Geoinformación de la Universitat Politècnica de València. Esta asignatura, que tiene 6 créditos ECTS, ( 3 teóricos y 3 prácticos) tiene como principal objetivo conseguir que los estudiantes sean expertos en las especificaciones de datos de la Directiva Europea Inspire. Es una asignatura técnicamente compleja y en la que los alumnos deben implementar una especificación de datos utilizando bases de datos 
espaciales. El contenido de la asignatura permite que el alumno llegue a ser capaz de integrarse en equipos multidisciplinares de empresas que trabajan en proyectos con datos geoespaciales, pero además tiene que facilitar al alumno habilidades de hablar en público, trabajar en equipo y liderar proyectos. Desde hace algunos años la UPV tiene 13 competencias transversales integradas en la evaluación y su objetivo es conseguir que todos sus egresados lleguen a alcanzarlas en unos niveles adecuados (ICE-UPV 2018). Esta asignatura es punto de control de las competencias de liderazgo y trabajo en grupo y de la competencia de comunicación efectiva y para evaluar parte de dichas competencias se utiliza la coevaluación y la autoevaluación.

El uso de la coevaluación y la autoevaluación en el contexto universitario ha sido estudiado por varios autores a lo largo de estos últimos años y según (Deeley 2014), (Quesada, Gómez y Cubero 2015) existen diversos beneficios en el uso de la coevaluación en entornos universitarios, ya que al utilizar estas técnicas hacemos que nuestros estudiantes mejoren sus habilidades relacionadas con evaluar a otros y especialmente potencian la capacidad de evaluarse a sí mismos. Cuando un estudiante se ve en la obligación moral de realizar juicios sobre los trabajos de sus compañeros (coevaluación) o sobre su propio trabajo (autoevaluación) implícitamente incorpora a su aprendizaje la ética y la lógica que lleva implícita la evaluación. Los estudiantes mejoran la competencia de comunicación efectiva y las habilidades para argumentar y negociar según (Quesada, García y Gómez 2016). Se incrementa la comunicación y la confianza mutua entre profesores y estudiantes (Quesada, Gómez y Cubero, 2015) y también permite que los estudiantes asuman diferentes roles en los trabajos realizados (Álvarez Valdivia, 2008).

El estudio realizado por Rodríguez, Ibarra, y Cubero (2016) en 10 universidades públicas españolas, analiza la percepción un tanto negativa que tienen los estudiantes sobre su capacidad evaluadora con la conclusión que sería necesario prepararlos en técnicas evaluación. Esto es fácil comprobarlo cuando se les pide que realicen una evaluación de sus compañeros, por ejemplo de una exposición oral en la que diferentes estudiantes desarrollan un tema y posteriormente se comprueba que no existe un reflejo de la realidad observada. Algunos estudiantes rellenan la rúbrica utilizada valorando con la máxima puntuación todos los apartados, o con valoraciones intermedias.

La experiencia conducida por Acedo, y Ruiz-Cabestre (2011) muestra resultados positivos al comparar el grado de objetividad entre las valoraciones que logran los estudiantes de sí mismos y de sus compañeros, mediante la autoevaluación y la evaluación de pares, respecto a las valoraciones hechas por el profesor, aunque como se ha mencionado anteriormente esto no siempre sucede. 
Y los autores García-Beltrán, Martínez, Jaén, Tapia (2016) describen cómo la autoevaluación de los estudiantes permite diagnosticar sus conocimientos previos y de esta forma se pueden orientar las diferentes actividades durante el desarrollo de la docencia de la asignatura.

Con la autoevaluación conseguimos hacer al estudiante responsable de su propio aprendizaje. Pasa de tener un papel pasivo a formar parte activa del proceso de aprendizaje. La autoevaluación ayuda a poder desarrollar estrategias para conseguir mejorar el autocontrol y la propia valoración de si mismo. Facilita el desarrollo de habilidades en grupo, favorece una actitud positiva hacia el aprendizaje, incrementa la cooperación entre los estudiantes y desarrolla el respeto a su autonomía.

\section{Metodología}

La metodología que se utiliza en la asignatura debe de garantizar poder evaluar al alumno en las dos competencias transversales de liderazgo y trabajo en grupo y de la comunicación efectiva, así como de las competencias específicas de la misma. El primer día de clase se especifica el calendario de las clases teóricas y prácticas que se van a llevar a cabo y de las actividades que se realizaran durante el cuatrimestre (Fig. 1)

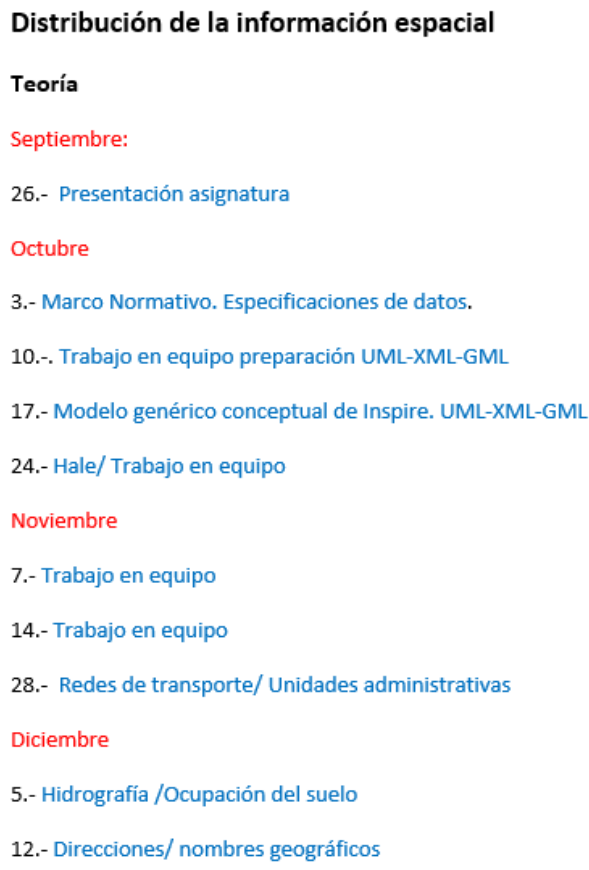

Fig. 1 Parte de la temporización de la asignatura 
Existen dos formas de valorar el trabajo en grupo y liderazgo; Todos los alumnos van a trabajar de la siguiente forma: Se generan ellos mismos grupos de 3 personas y eligen de todos las presentaciones técnicas de formatos exigidos por el Open Geospatial Consortiun para trabajar con información espacial que tienen disponibles en el PoliformaT la que quieren realizar. Por otro lado se dividen en 6 grupos generados desde el primer día por el profesor y se les asigna la especificación técnica que debe realizar para exponerla en clase el día especificado en la temporización.

Como se puede observar en la Fig. 1, se realizan sesiones de trabajo presenciales y al finalizar la clase los estudiantes generan una evidencia de la reunión. Dichas evidencias son actas de las sesiones de trabajo donde además de las conclusiones obtenidas en la sesión se debe proporcionar el orden del día y la firma de los asistentes a dicha reunión de trabajo.

Dentro del Poliformat existen varios apartados en los que se apoya esta asignatura. Los estudiantes deberán utilizar los chats para comunicarse entre ellos y si se programan reuniones virtuales, se deberá realizar una convocatoria con 24 horas de antelación al grupo y al profesor para, si es necesario, el profesor pueda conectarse a la misma y realizar una valoración de los roles utilizados durante la reunión.

Sistemas de evaluación y calificación.

En la guía docente de la asignatura aparece el sistema de evaluación que se va a emplear y es conocido desde el principio por todos los estudiantes. Al tener que evaluar competencias técnicas y transversales a la vez, se opta por utilizar diversas técnicas de aprendizaje en las que consigamos una evaluación formativa y continua. Se pretende motivar al alumno y de esta forma aumentar su aprendizaje y rendimiento académico.

Uno de los principales problemas que tiene este tipo de evaluación es que es necesario una buena planificación, organización y dedicación a concretar los criterios de evaluación y calificación para poder garantizar una alta fiabilidad del instrumento de evaluación diseñado.

La técnica de coevaluación es utilizada para evaluar la competencia de comunicación oral. Todos los estudiantes necesitan hacer dos exposiciones públicas para conseguirlo y son sus compañeros los que les evalúan utilizando la rúbrica que se presenta en la Fig. 2. 


\begin{tabular}{|c|c|c|c|c|}
\hline & 4 & 3 & 2 & 1 \\
\hline Pronunciación & $\begin{array}{l}\text { Habla despacio y } \\
\text { claro y vocaliza } \\
\text { bien }\end{array}$ & $\begin{array}{l}\text { La mayoría del } \\
\text { tiempo habla } \\
\text { despacio y con } \\
\text { claridad }\end{array}$ & $\begin{array}{l}\text { Unas veces habla } \\
\text { despacio y con } \\
\text { claridad y otras es } \\
\text { dificil de entender }\end{array}$ & $\begin{array}{l}\text { Habla rápido o } \\
\text { presenta espacios } \\
\text { sin hablar. Con } \\
\text { muletillas }\end{array}$ \\
\hline Volumen & $\begin{array}{l}\text { Adecuado y lo } \\
\text { suficientemente alto } \\
\text { cuando enfatiza lo } \\
\text { importante }\end{array}$ & $\begin{array}{l}\text { Levanta } \\
\text { demasiado la } \\
\text { voz todo el } \\
\text { tiempo. }\end{array}$ & $\begin{array}{l}\text { Habla demasiado } \\
\text { bajo al exponer y } \\
\text { de forma } \\
\text { monótona. }\end{array}$ & $\begin{array}{l}\text { El volumen es } \\
\text { muy débil, casi } \\
\text { no se le oye }\end{array}$ \\
\hline $\begin{array}{l}\text { Postura del } \\
\text { cuerpo/contacto } \\
\text { visual }\end{array}$ & $\begin{array}{l}\text { Postura y gestos son } \\
\text { muy adecuados. } \\
\text { Mira a todos los } \\
\text { compañeros con } \\
\text { naturalidad }\end{array}$ & $\begin{array}{l}\text { La postura y los } \\
\text { gestos son } \\
\text { adecuados pero, } \\
\text { suele mirar sólo } \\
\text { a alguna parte de } \\
\text { la audiencia. }\end{array}$ & $\begin{array}{l}\text { En ocasiones le da } \\
\text { la espalda a sus } \\
\text { compañeros. } \\
\text { Algunas veces } \\
\text { mantiene la } \\
\text { postura adecuada. }\end{array}$ & $\begin{array}{l}\text { No mantiene la } \\
\text { postura apropiada } \\
\text { y la mayoría de } \\
\text { las veces no mira } \\
\text { a sus compañeros. }\end{array}$ \\
\hline Contenido & $\begin{array}{l}\text { Demuestra un } \\
\text { completo } \\
\text { entendimiento del } \\
\text { tema. }\end{array}$ & $\begin{array}{l}\text { Demuestra un } \\
\text { buen } \\
\text { entendimiento } \\
\text { del tema. }\end{array}$ & $\begin{array}{l}\text { Demuestra } \\
\text { entendimiento de } \\
\text { partes del tema. }\end{array}$ & $\begin{array}{l}\text { No parece } \\
\text { entender muy } \\
\text { bien el tema. }\end{array}$ \\
\hline Vocabulario & $\begin{array}{l}\text { Usa vocabulario } \\
\text { técnico que } \\
\text { enriquece a la } \\
\text { audiencia. }\end{array}$ & $\begin{array}{l}\text { Usa vocabulario } \\
\text { técnico e incluye } \\
\text { algunas palabras } \\
\text { nuevas para la } \\
\text { mayor parte de } \\
\text { la audiencia }\end{array}$ & $\begin{array}{l}\text { Usa vocabulario } \\
\text { apropiado y } \\
\text { conocido por la } \\
\text { audiencia. }\end{array}$ & $\begin{array}{l}\text { No usa } \\
\text { vocabulario } \\
\text { técnico y repite } \\
\text { muchas veces las } \\
\text { mismas palabras }\end{array}$ \\
\hline $\begin{array}{l}\text { Comprensión/ } \\
\text { secuenciación }\end{array}$ & $\begin{array}{l}\text { Presenta una } \\
\text { conexión fluida, } \\
\text { sigue el orden } \\
\text { lógico en la } \\
\text { exposición y } \\
\text { contesta las } \\
\text { preguntas } \\
\text { planteadas. }\end{array}$ & $\begin{array}{l}\text { Puede contestar } \\
\text { algunas } \\
\text { preguntas y la } \\
\text { conexión entre } \\
\text { las diapositivas } \\
\text { es bastante } \\
\text { buena. }\end{array}$ & $\begin{array}{l}\text { Realiza muy poco } \\
\text { la conexión entre } \\
\text { las diapositivas y } \\
\text { contesta muy poco } \\
\text { a las preguntas. }\end{array}$ & $\begin{array}{l}\text { La exposición } \\
\text { carece de orden. } \\
\text { No conecta las } \\
\text { diapositivas y no } \\
\text { puede contestar } \\
\text { las preguntas } \\
\text { planteadas. }\end{array}$ \\
\hline
\end{tabular}

Fig 2: Rubrica utilizada para la coevaluación. 
La coevaluación y autoevaluación en la asignatura de Distribución de la Información Espacial del $M U I G G$ en la UPV

En cada sesión, los estudiantes disponen de la tabla 1 y deben valorar a todos los compañeros que están haciendo la presentación oral.

Tabla 1: Tabla utilizada por los estudiantes

\begin{tabular}{|l|l|l|l|l|}
\hline Alumno: & Nombre1 & Nombre2 & Nombre3 & Nombre4 \\
\hline Pronunciación & & & \\
\hline Volumen & & & \\
\hline Postura del cuerpo/ contacto & & \\
visual & & & \\
\hline Contenido & & & \\
\hline Vocabulario & & & \\
\hline Comprensión/secuenciación & & & \\
\hline
\end{tabular}

En la tabla 2 se presentan los resultados obtenidos en un grupo de 15 alumnos

Tabla 2: Resultados de la coevaluación

\begin{tabular}{|c|c|c|c|c|c|c|c|c|c|c|c|c|c|c|c|c|c|c|}
\hline & alumno1 & alumno2 & alumno3 & alumno4 & alumno5 & alumno6 & alumno7 & alumno8 & alumno9 & alumno10 & alumno11 & alumno12 & alumno13 & 3 alumno14 & 4 alumno 15 & alumno16 & & autoevaluacio \\
\hline alumno1 & & & & & & 4 & 4 & 4 & 3 & 4 & 4 & 4 & 3 & 4 & 4 & 4 & 4 & 0.9 \\
\hline alumno2 & & & & & & 4 & 4 & 4 & 4 & 4 & 4 & 4 & 4 & 4 & 4 & 3 & 4 & 0,9 \\
\hline alumno3 & & & & & & 4 & 4 & 4 & 3 & 4 & 4 & 4 & 4 & 4 & 4 & 3 & 4 & 0,9 \\
\hline alumno4 & & & & & & 3 & 4 & 4 & 3 & 3 & 3 & 3 & 3 & \begin{tabular}{l|l}
3 \\
\end{tabular} & 4 & 3 & 3 & 0,6 \\
\hline alumno5 & & & & & & 3 & 4 & 4 & 3 & 4 & 4 & 4 & 4 & 4 & 4 & 4 & 4 & 0,9 \\
\hline alumno6 & 4 & 4 & 4 & 4 & & & & & & & 4 & 4 & 4 & 4 & 4 & 3 & 4 & 0,9 \\
\hline alumno7 & 4 & 4 & 3 & 4 & & & & & & & 4 & 3 & 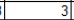 & 4 & 4 & 4 & 4 & 0,9 \\
\hline alumno8 & 3 & 3 & 3 & 3 & & & & & & & & 3 & 3 & 3 & 4 & 3 & 4 & 0,7 \\
\hline alumno9 & 4 & 4 & 3 & 4 & & & & & & & 4 & 4 & 3 & 4 & 4 & 4 & 4 & 0,9 \\
\hline alumno10 & 3 & 4 & 4 & 4 & & & & & & & 3 & 4 & 3 & 3 & 4 & 4 & 4 & 0,9 \\
\hline alumno11 & 4 & 4 & 4 & 4 & & 4 & & 3 & 3 & 4 & & & & & & & 4 & 0,9 \\
\hline alumno12 & 4 & 4 & 3 & 4 & & 3 & & 3 & 3 & 4 & 4 & & & & & & 3 & 0,7 \\
\hline alumno13 & 3 & 3 & 3 & 3 & & 3 & & 3 & 3 & 3 & & & & & & & 3 & 0,7 \\
\hline alumno14 & 4 & 4 & 3 & 3 & & 3 & & 3 & 3 & 3 & & & & & & & 3 & 0,7 \\
\hline alumno15 & 3 & 4 & 3 & 4 & & 3 & 3 & 3 & 3 & 4 & 4 & & & & & & 3 & 0,5 \\
\hline alumno16 & 2 & 3 & 2 & 3 & & 2 & 2 & 3 & 2 & 2 & 2 & & & & & & 2 & 0,4 \\
\hline
\end{tabular}

Durante la última semana de clase el estudiante realiza un autoevaluación donde debe valorar 10 ítems que se resumen en la fig. 3. El estudiante valora las aportaciones que ha realizado durante el desarrollo de la asignatura, su trabajo de forma individual y el trabajo realizado en equipo. Se valora también las relaciones entre los miembros del grupo y el tiempo que se ha dedicado a las diferentes actividades. Es recomendable el haber ido a tutorías con el profesor. Para finalizar la autoevaluación se debe valorar la satisfacción personal y una valoración global. 


\section{Autoevaluacio̊n gene̊rica}
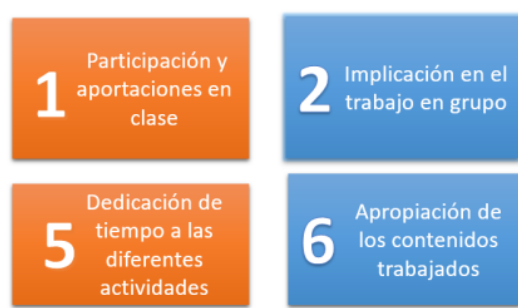

(9) Satisfacción

personal
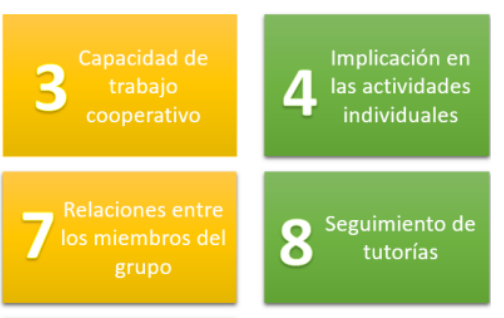

10 Valoración

global

Fig. 3 Autoevaluación generica

La asignatura persigue conseguir competencias para que los estudiantes consigan responsabilizarse de su propio aprendizaje, utilizando categorías técnicas, metodológicas, participativas y personales
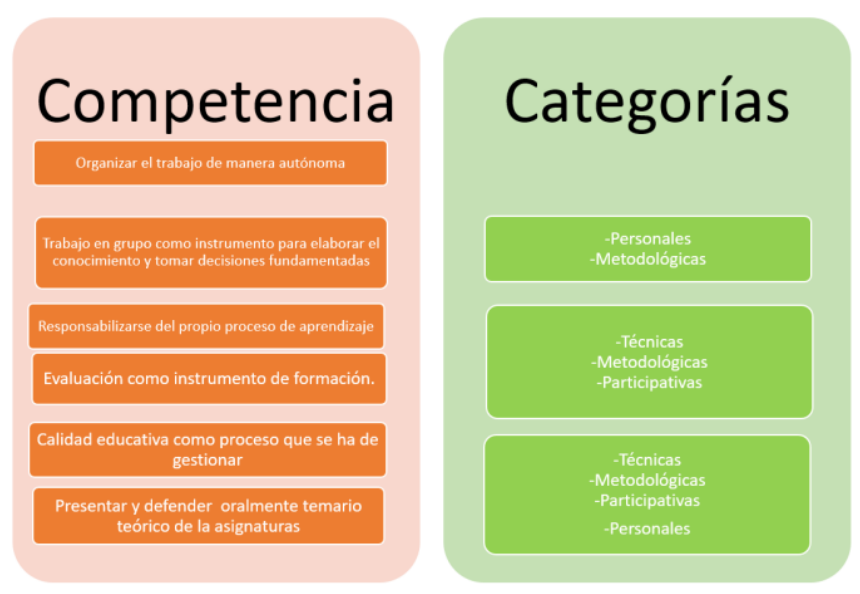

Fig. 4 Competencias y categorías de la asignatura 


\section{Conclusiones}

Como conclusiones obtenidas durante estos dos últimos cursos académicos se puede considerar que la evaluación formativa y continua, realizada en esta asignatura incrementa los resultados académicos en los estudiantes. Cada vez son más conscientes del proceso de aprendizaje gracias a que existe un seguimiento más individualizado por parte del profesor y un incremento en la asistencia a tutorías por parte de los estudiantes.

Los alumnos presentan mayor aceptación de sus calificaciones y son conscientes del trabajo que lleva una evaluación por parte del profesorado y consideran más justas las notas obtenidas.

Se detectan casos, sobretodo en estudiantes erasmus, que cuando utilizan la rúbrica, valoran todos los apartados con la misma puntuación y muchas veces no refleja la realidad. Para el próximo curso académico se pretende realizar una primera valoración al profesor para que sirva de ejemplo cuando se realicen las exposiciones públicas y de esa forma incidir en la misma conclusión que los autores Rodríguez, Ibarra, y Cubero (2016) en el que los estudiantes necesitan preparación en técnicas de evaluación.

Creemos que es interesante para el siguiente curso académico, realizar la coevaluación con parejas no recíprocas, esto ayudaría a desarrollar las competencias en comunicación efectiva, competencias de análisis crítico, incrementaría la responsabilidad en los estudiantes y su participación con más compañeros.

Por ultimo, agradecemos a la Universitat Politècnica de València. la posibilidad de poder difundir los trabajos realizados en la docencia de las asignaturas y en concreto en las evaluaciones de las competencias transversales, que para esta misma asignatura se presentaron las conclusiones obtenidas en la evaluación de la comunicicaciñon efectiva y el trabajo en equipo y liderazgo en el congreso IN-RED 2018.

\section{Referencias}

Wolchik, S. A., West, S. G., Sandler, I. N., Tein, J., Coatsworth, D., Lengua, L., et al. (2000). An experimental evaluation of theory-based mother and mother-child programs for children of divorce. Journal of Counseling and Clinical Psychology, 68, 843-856.

Acedo, M. Á., \& Ruiz-Cabestre, F. J. (2011). Una experiencia sobre la evaluación autónoma o participativa: autoevaluación y evaluación por los compañeros. Arbor, 187(Extra_3), 183-188.

Coll-Aliaga, Eloína; Martínez Llario, José Carlos (2018). Evaluación de las competencias de trabajo en equipo y liderazgo y la comunicación efectiva en la asignatura de 
Distribución de la información espacial del MUIGG. IV Congreso Nacional de Innovación Educativa y Docencia en Red (IN-RED 2018). (1 - 8). Valencia, España: Editorial Universitat Politècnica de València. doi: 10.4995/INRED2018.2018.8887

Deeley, S. (2014). Summative co-assessment: A deep learning approach to enhancing employability skills and attributes. Active Learning in Higher Education, 15(1) 39-51. https://doi.org/10.1177/1469787413514649.

Garcia-Beltrán, Ángel, Martínez Raquel, Jaén José-Alberto, Tapia Santiago (2016). La autoevaluación como actividad docente en entornos virtuales de aprendizaje/enseñanza. RED. Revista de Educación a Distancia, 50.

\section{http://revistas.um.es/red/article/view/272241/198741}

ICE-UPV (2018). Portal de competencias transversales de la Universitat Politècnica de València. http://www.upv.es/contenidos/COMPTRAN/ [Online; acceso 06-julio-2018].

https:/www.upv.es/entidades/ICE/info/U0724624.pdf

Quesada, V., García Jiménez, E. y Gómez Ruiz, M. A. (2016). Student participation in assessment processes: A way forward. En E. Cano y G. Ion (Eds.), Innovative practices for higher education assessment and measurement (pp. 228-249). Hershey, PE: IGI Global.

Quesada Serra, V., Gómez Ruiz, M. A. y Cubero Ibáñez, J. (2015). La evaluación colaborativa en Educación Superior: Descripción de una experiencia con alumnos de primer curso. Universitat de Girona (Ed.), V Congreso Internacional UNIVEST'15. Los retos de mejorar la evaluación (pp. 562-566). Girona: Institut de Ciències de l'Educació

Quesada Serra, V., Rodríguez Gómez, G. e Ibarra Sáiz, M. S. (2016). What are we missing? Spanish lecturers' perceptions of their assessment practices. Innovations in Education and Teaching International, 53(1), 48-59. doi:10.1080/14703297.2014.930353..

Rodríguez Gómez, G.; Ibarra Sáiz, M.S. y Cubero Ibáñez, J. (2016). Competencias básicas relacionadas con la evaluación. Un estudio sobre la percepción de los estudiantes universitarios. Educación XX1 